\title{
Vaccinia Virus DD-CDSR
}

National Cancer Institute

\section{Source}

National Cancer Institute. Vaccinia Virus DD-CDSR. NCI Thesaurus. Code C74089.

\begin{abstract}
A highly tumor-selective vaccinia virus (vv) with an engineered double deletion (DD) of the thymidine kinase (tk) and vaccinia growth factor genes and additions of both a cytosine deaminase (CD) gene and a somatostatin receptor (SR) gene with potential oncolytic viral activity. The tk and vaccinia growth factor gene deletions in intratumorally administered vaccinia virus (VVDD-CDSR) help to restrict its replication and cytolytic activity to tumor cells with large nucleotide pools and tumor cells with activation of the EGFR-Ras pathway. Addition of the CD gene to the viral genome allows control of oncolytic viral infection through the administration of the prodrug 5-fluorocytosine (5-FC), converted by CD to the antimetabolite 5-fluorouracil (5-FU) in cells infected with this agent. Addition of the SR gene allows anatomical localization of vaccinia virus (VVDD-CDSR) through the use of octreotide scintigraphy.
\end{abstract}

